\title{
Outage probability of power splitting SWIPT two-way relay networks in Nakagami-m fading
}

\author{
Shaohong Zhong ${ }^{1,2}$, Huajun Huang ${ }^{2}$ and Renfa Li ${ }^{\text {* }}$
}

\begin{abstract}
This paper investigates the outage probability of an energy harvesting $(\mathrm{EH})$ relay-aided cooperative network, where a source node transmits information to its destination node with the help of an energy harvesting cooperative node. For such a system, we derive an explicit closed-form expression of outage probability over Nakagami-m fading channels for both amplify-and-forward (AF) and decode-and-forward (DF) relay protocols, and we verify the explicit closed-form expressions of outage probability with the Monte Carlo method. It is shown that the simulation results match well with the numerical ones. From the numerical analysis and simulation results, it can be observed that the system parameters have great impact on both AF and DF relay systems. For the DF system, with the increment of the power splitting ratio, the system outage probability decreases, while for the AF system, with the increment of the power splitting ratio, it first increases and then decreases. Besides, for both DF and AF systems, when the relay is placed relatively closer to the source, better outage performance will be achieved.
\end{abstract}

Keywords: Energy harvesting, Nakagami-m fading channel, Outage probability, Relay-aided cooperative networks, Power splitting

\section{Introduction}

Nowadays, Energy harvesting has appeared as a promising approach to prolong lifetime of energy constrained wireless communication system [1-4], which is usually equipped with replacing or recharging batteries. For example, in wireless sensor networks, if a sensor is depleted of energy, it will be out of work. And replacing or recharging energy may be unavailable, especially when a sensor is embedded in building structures or inside human bodies. Earlier, the energy harvesting technologies mostly relied on external and traditional energy sources such as solar, wind, and vibration. However, the application range of the traditional energy harvesting technologies is limited because of the environment uncertainty, weather dependence.

Very recently, simultaneous wireless information and power transfer (SWIPT) has been an exciting new way

${ }^{*}$ Correspondence: rfli@vip.sina.cn

${ }^{1}$ College of Computer Science and Electronic Engineering and the Key

Laboratory for Embedded and Network Computing, Hunan University, 410082

Changsha, People's Republic of China

Full list of author information is available at the end of the article to provide stale energy to wireless communication where the receiver is able to harvest energy and decode information from the received signals [5-11]. In [5], the authors described the basic idea about the wireless information and power transfer from information theoretical perspective, and in [6], the authors proposed a general receiver architecture with separated information decoding and energy harvesting receiver for SWIPT for practical applications.

Following these pioneering works, plenty of works have been done for various wireless networks, including cooperative relaying network [12-16], power allocation strategies [17-19], resource scheduling [20, 21], and multiple-input multiple-out (MIMO) system [22-24] or multiple-input single-output (MISO) [25, 26]. In [12], the authors analyzed the system maximal throughput for the time switching and power splitting protocols in AF relaying networks. The authors in [13] investigated the outage performance of the relay network over rayleigh fading channels where both AF and DF protocols were considered. In [14], the authors investigated the relay selection for SWIPT systems. In [17], the authors investigated the

\section{Springer Open}

(c) The Author(s). 2018 Open Access This article is distributed under the terms of the Creative Commons Attribution 4.0 International License (http://creativecommons.org/licenses/by/4.0/), which permits unrestricted use, distribution, and reproduction in any medium, provided you give appropriate credit to the original author(s) and the source, provide a link to the Creative Commons license, and indicate if changes were made. 
multi-user cooperative networks where how to distribute the harvested energy among the multiple users was studied. In [18], the authors discussed two types of power allocation policies for non-orthogonal multiple access (NOMA) system. In [20], the author proposed a greedy clustering algorithms to reduce the hardware cost of the PS scheme. In [22], a non-regenerative MIMO orthogonal frequency-division multiplexing (OFDM) relaying system was investigated, and the maximal achievable information rates of two protocols, time swithing-based relaying and power splitting-based relaying, were explored. In [23], the authors focused on quality-of-service-constrained energy efficient optimization in MIMO SWIPT systems via joint antenna selection and spatial switching. In [25] and [26], the secrecy performance of channel uncertainties and imperfect channel state information was studied for multiple-input and multiple-output SWIPT system.

In this paper, we focus on a two-way relay SWIPT system over Nakagami-m fading, which consists of a source-destination (S-D) pair and a relay node $R$ embedded power splitting (PS) (see Fig. 1). We investigate the effect of SWIPT on the outage probability and the impact of the ambient environment energy harvesting and the RF energy harvesting on the system outage probability. In detail, a closed-form expression of outage probability considering AF/DF relaying schemes at node $R$ to decode and forward the received signals to node $D$ is derived.

The contributions are expressed as below: at first, we derive a closed-form expressions of outage probability at two-way relay cooperation group for AF and DF relaying schemes with Nakagami-m fading channels respectively. Secondly, we simulate the outage probability of two-way relay SWIPT systems with Monte Carlo method; at the same time, we consider the impact of power splitting fraction, the position of the relay node between the source node and destination node, the buffer energy capacity of relay node harvesting from ambient environment, etc. Finally, we give the comparison of system operation time of numerical analysis and Monte Carlo method.

The rest of the paper is organized as follows. Section 2 reports about model of the system. Section 3 presents the outage probability analysis. Section 4 presents our numerical result and validates the analytical result through Monte Carlo simulations. Finally, a conclusion will be drawn in Section 5.

\section{System model}

In the first section, we assumed a simplest cooperation group including a source-destination $(S-D)$ pair, and a relay node $R$. All links experience independent and identically Nakagami-m fading. In the first phase, the received signals at the information receiver of $\mathrm{R}$ and $\mathrm{D}$ in downlink phase are expressed as

$$
\begin{aligned}
y_{R} & =\sqrt{\rho}\left(\sqrt{P_{s}} h_{s r} X+n_{R}\right)+z_{R} \\
y_{D 1} & =\sqrt{P_{s}} h_{s d} x+n_{D}
\end{aligned}
$$

where $P_{i}(i \in(S, R))$ is the transmit power at node $i, x$ denotes the transmitted symbols from node $S, h_{i j}(i, j \in$ $S, R, D)$ is the link channel gain between node $i$ and $j$, $n_{i}(i \in D, R)$ denotes the independent complex Gaussian noise at the information receiver of node $R$ and $D$ with zero means and a same variance, and $N_{0}$ and $z_{R}$ are the signal processing noise by the information decoder at node $R$ followed by additional white Gaussian noise with zero means and variance $\sigma^{2}$.

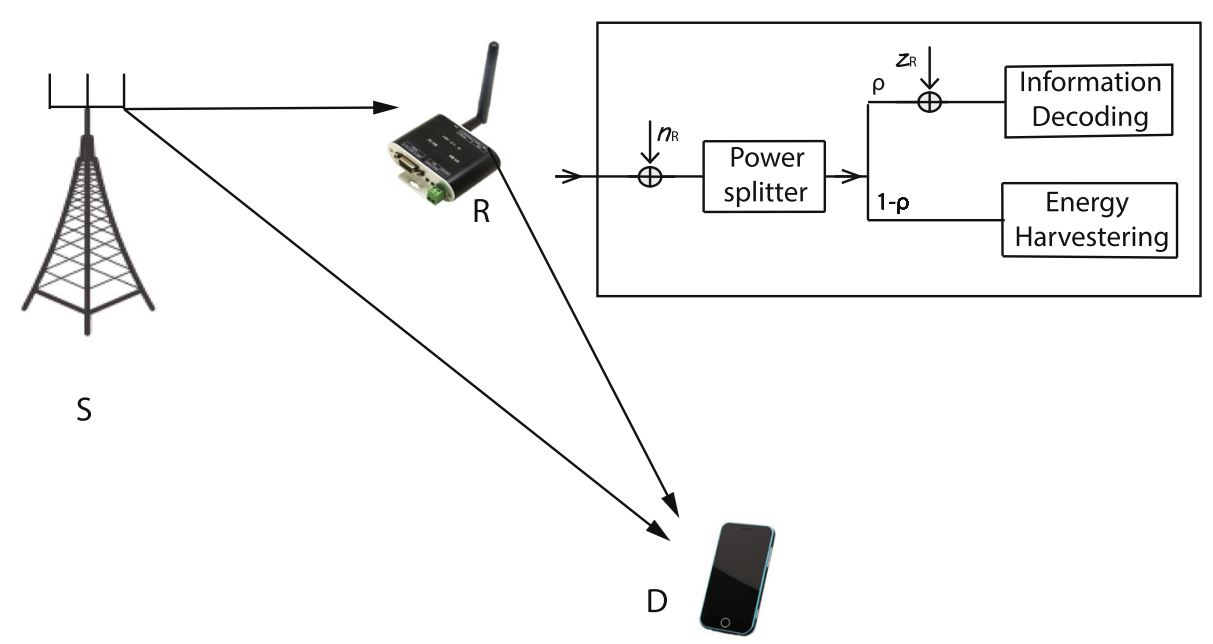

Fig. 1 A system structure for energy harvesting relay-aided cooperative network with a power splitter relay node 
So, the SNR of the received signal at information receiver of $R$ and $D$ can be expressed as

$$
\begin{aligned}
& \gamma_{s r}=\frac{\rho P_{s}\left|h_{s r}\right|^{2}}{\left.\left(\rho N_{0}+\sigma^{2}\right)(\alpha d)^{2}\right)} \\
& \gamma_{s d}=\frac{P_{s}\left|h_{s d}\right|^{2}}{N_{0} d^{2}}
\end{aligned}
$$

where $\rho$ denotes the ratio of power splitter, $\alpha$ denotes the ratio of the distance of the relay node position departing from the source node $S$ to the distance $d$ between the source node $S$ and the destination node $D$.

The probability density function (PDF) of $\left|h_{s r}\right|^{2}$ and $\left|h_{s d}\right|^{2}$ can be given as

$$
\begin{aligned}
& p\left(\left|h_{s r}\right|^{2}\right)=\frac{m_{s r}{ }^{m_{s r}} \gamma_{s r} m_{s r}-1}{{\overline{\gamma_{s r}}}^{m_{s r}} \Gamma\left(m_{s r}\right)} \exp \left(-\frac{m_{s r}}{\overline{\gamma_{s r}}} \gamma_{s r}\right) \\
& p\left(\left|h_{s d}\right|^{2}\right)=\frac{m_{s d}{ }^{m_{s d}} \gamma_{s d}{ }^{m_{s d}-1}}{\overline{\gamma_{s d}} m_{s d}^{m_{d}} \Gamma\left(m_{s d}\right)} \exp \left(-\frac{m_{s d}}{\overline{\gamma_{s d}}} \gamma_{s d}\right)
\end{aligned}
$$

where $m_{s d}$ and $m_{s r}$ denote that the Nakagami-m fading parameter at the respective hop. $\Gamma($.$) stands for the$ Gamma function [27], and average SNR $\left(\overline{\gamma_{s r}}\right.$ and $\left.\overline{\gamma_{s d}}\right)$ of received signal at relay and destination $[28,29]$ can be written as

$$
\begin{aligned}
& \overline{\gamma_{s r}}=\rho P_{s} \frac{E\left(\left|h_{s r}\right|^{2}\right)}{\left(\rho N_{0}+\sigma^{2}\right)(\alpha d)^{2}}=\rho P_{s} \frac{E_{h 1}}{\left.\left(\rho N_{0}+\sigma^{2}\right)(\alpha d)^{2}\right)} \\
& \overline{\gamma_{s d}}=P_{s} \frac{E\left(\left|h_{s d}\right|^{2}\right)}{N_{0}}=P_{s} \frac{E_{h 2}}{N_{0} d^{2}}
\end{aligned}
$$

\section{Outage analysis}

\subsection{DF scheme outage analysis}

In this second phase, if $R$ decides to forward the detected symbols, $X_{r}$, to $D$ after regenerating, the received signal at $D$ is

$$
y_{D 2 d f}=\sqrt{P_{r}} h_{r d} X_{r}+n_{D}
$$

where $P_{r}=(1-\rho) P_{R F}+E_{\max }$, where $P_{R F}$ denotes the energy harvested by relay node through RF and $E_{\max }$ denotes the natural energy harvested by relay node from ambient environment (e.g., solar energy, wind energy).

Accordingly, the SNR of the received signal at $D$ can be expressed as

$$
\gamma_{r d}=\frac{P_{r}\left|h_{r d}\right|^{2}}{N_{0}(1-\alpha d)^{2}}
$$

The probability density function (PDF) of $\left|h_{r d}\right|^{2}$ can be given as

$$
p\left(\left|h_{r d}\right|^{2}\right)=\frac{m_{r d} m_{r d} \gamma_{r d} m_{r d}-1}{\overline{\gamma_{r d}} m_{r d} \Gamma\left(m_{r d}\right)} \exp \left(-\frac{m_{r d}}{\gamma_{r d}} \gamma_{r d}\right)
$$

where the average SNR of the received signal at destination can be written as

$$
\overline{\gamma_{r d}}=P_{r} \frac{E\left(\left|h_{r d}\right|^{2}\right)}{N_{0}(1-\alpha d)^{2}}=P_{r} \frac{E_{h 3}}{N_{0}(1-\alpha d)^{2}}
$$

The outage probability of the consider system for the DF scheme can be expressed as

$$
\begin{gathered}
P_{\text {out }}\left(\gamma_{t h}\right)=P\left[\gamma_{s r}>\gamma_{t h}\right] P\left[\gamma_{d} \leq \gamma_{t h} \mid \gamma_{s r}>\gamma_{t h}\right] \\
+P\left[\gamma_{s r} \leq \gamma_{t h}\right] P\left[\gamma_{D 1} \leq \gamma_{t h}\right]
\end{gathered}
$$

The probability with the SNR of $S-R$ link $\gamma_{s r}$ can be expressed as

$$
\begin{aligned}
P\left[\gamma_{s r} \leq \gamma_{t h}\right] & =\int_{0}^{\gamma_{t h}} \frac{m_{s r} m_{s r} \gamma_{s r} m_{s r}-1}{\overline{\gamma_{s r}}{ }^{m_{s r}} \Gamma\left(m_{s r}\right)} \exp \left(-\frac{m_{s r}}{\overline{\gamma_{s r}}} \gamma_{s r}\right) d \gamma_{s r} \\
& =1-\exp \left(-\frac{m_{s r}}{\overline{\gamma_{s r}}} \gamma_{t h}\right) \sum_{n=0}^{m_{s r}-1}\left(\frac{m_{s r} \gamma_{t h}}{\overline{\gamma_{s r}}}\right)^{n} \frac{1}{n !}
\end{aligned}
$$

Similarly, the probability with the SNR of $S-D$ link $\gamma_{s d}$ can be expressed as

$$
\begin{aligned}
P\left[\gamma_{s d} \leq \gamma_{t h}\right] & =\int_{0}^{\gamma_{t h}} \frac{m_{s d}^{m_{s d}} \gamma_{s d} m_{s d}-1}{\overline{\gamma_{s d}} m_{s d} \Gamma\left(m_{s d}\right)} \exp \left(-\frac{m_{s d}}{\overline{\gamma_{s d}}} \gamma_{s d}\right) d \gamma_{s d} \\
& =1-\exp \left(-\frac{m_{s d}}{\overline{\gamma_{s d}}} \gamma_{t h}\right) \sum_{n=0}^{m_{s d}-1}\left(\frac{m_{s d} \gamma_{t h}}{\overline{\gamma_{s d}}}\right)^{n} \frac{1}{n !}
\end{aligned}
$$

The probability with the SNR of the combined signal at D, $\gamma_{D}$, can be expressed as

$$
\begin{aligned}
P\left[\gamma_{d} \leq \gamma_{t h} \mid \gamma_{s r}>\gamma_{t h}\right]= & P\left[\gamma_{D} \leq \gamma_{t h}\right]=P\left[\max \left\{\gamma_{D 1}, \gamma_{D 2}\right\} \leq \gamma_{t h}\right] \\
= & {\left[1-\exp \left(-\frac{m_{s d}}{\overline{\gamma_{s d}}} \gamma_{t h}\right) \sum_{n=0}^{m_{s d}-1}\left(\frac{m_{s d} \gamma_{t h}}{\overline{\gamma_{s d}}}\right)^{n} \frac{1}{n !}\right] } \\
& \times\left[1-\exp \left(-\frac{m_{r d}}{\overline{\gamma_{r d}}} \gamma_{t h}\right) \sum_{n=0}^{m_{r d}-1}\left(\frac{m_{r d} \gamma_{t h}}{\overline{\gamma_{r d}}}\right)^{n} \frac{1}{n !}\right]
\end{aligned}
$$


Therefore, the outage probability can be obtained by substituting (14), (15), and (16) into (13) as

$$
\begin{aligned}
P_{\text {out }}^{(d f)}\left(\gamma_{t h}\right)= & \exp \left(-\frac{m_{s r}}{\overline{\gamma_{s r}}} \gamma_{t h}\right) \sum_{n=0}^{m_{s r}-1}\left(\frac{m_{s r} \gamma_{t h}}{\overline{\gamma_{s r}}}\right)^{n} \frac{1}{n !} \\
& \times\left[1-\exp \left(-\frac{m_{s d}}{\overline{\gamma_{s d}}} \gamma_{t h}\right) \sum_{n=0}^{m_{s d}-1}\left(\frac{m_{s d} \gamma_{t h}}{\overline{\gamma_{s d}}}\right)^{n} \frac{1}{n !}\right] \\
& \times\left[1-\exp \left(-\frac{m_{r d}}{\overline{\gamma_{r d}}} \gamma_{t h}\right) \sum_{n=0}^{m_{r d}-1}\left(\frac{m_{r d} \gamma_{t h}}{\overline{\gamma_{r d}}}\right)^{n} \frac{1}{n !}\right] \\
& +\left[1-\exp \left(-\frac{m_{s r}}{\overline{\gamma_{s r}}} \gamma_{t h}\right) \sum_{n=0}^{m_{s r}-1}\left(\frac{m_{s r} \gamma_{t h}}{\overline{\gamma_{s r}}}\right)^{n} \frac{1}{n !}\right] \\
& \times\left[1-\exp \left(-\frac{m_{s d}}{\overline{\gamma_{s d}}} \gamma_{t h}\right) \sum_{n=0}^{m_{s d}-1}\left(\frac{m_{s d} \gamma_{t h}}{\overline{\gamma_{s d}}}\right)^{n} \frac{1}{n !}\right]
\end{aligned}
$$

\subsection{AF scheme outage analysis}

In this second phase, if the node $R$ decides to forward the detected symbols, $X_{r}$, to the node $D$ after amplifying, the received signal at node $D$ can be expressed as

$$
\begin{aligned}
y_{D 2 a f} & =G h_{r d} y_{R}+n_{D} \\
& =G h_{r d}\left[\sqrt{\rho}\left(\sqrt{P_{s}} h_{s r} X+n_{R}\right)+z_{R}\right]+n_{D}
\end{aligned}
$$

where $G=\frac{\sqrt{P_{r}}}{\sqrt{P_{s}\left|h_{s r}\right|^{2}+N_{0}}}$ is the amplifying factor at $R$. Therefore, the SNR of the received signal at $D$ can be expressed as

$$
\begin{aligned}
\gamma_{r d 2} & =\frac{\frac{P_{s} P_{r} \rho\left|h_{r d}\right|^{2}\left|h_{s r}\right|^{2}}{P_{s}\left|h_{s r}\right|^{2}+N_{0}}}{\frac{P_{s}\left|h_{r d}\right|^{2}}{\left.P_{s} h_{s r}\right|^{2}+N_{0}}\left(\rho N_{0}+\sigma^{2}\right)+N_{0}} \\
& =\frac{\frac{\rho P_{s}\left|h_{s r}\right|^{2}}{\rho N_{0}+\sigma^{2}} \frac{P_{r}\left|h_{r d}\right|^{2}}{N_{0}}}{\frac{1}{\rho} \frac{\rho P_{s}\left|h_{s r}\right|^{2}}{\rho N_{0}+\sigma^{2}}+\frac{P_{r}\left|h_{r d}\right|^{2}}{N_{0}}+\frac{N_{0}}{\rho N_{0}+\sigma^{2}}} \\
& =\frac{\gamma_{s r} \gamma_{r d}}{a \gamma_{s r}+\gamma_{r d}+b}=\frac{\gamma_{1} \gamma_{2}}{a \gamma_{1}+\gamma_{2}+b}
\end{aligned}
$$

where $a=\frac{1}{\rho}, b=\frac{N_{0}}{\rho N_{0}+\sigma^{2}}, \gamma_{s r}=\gamma_{1}$, and $\gamma_{r d}=\gamma_{2}$. Let $P_{s r d}^{\text {(out) }}$ denotes the outage probability of $\gamma_{r d 2}$, so it can be expressed as

$$
\begin{aligned}
& P_{\text {srd }}^{\text {(out) }}=P\left[\gamma_{r d 2} \leq \gamma_{t h}\right]=\int_{0}^{\gamma_{t h}} P\left[\gamma_{2} \geq \frac{(a x+b) \gamma_{t h}}{x-\gamma_{t h}} \mid \gamma_{1}\right] \cdot f_{\gamma_{1}}(x) d x \\
& +\int_{\gamma_{\text {th }}}^{\infty} P\left[\gamma_{2} \leq \frac{(a x+b) \gamma_{t h}}{x-\gamma_{\text {th }}} \mid \gamma_{1}\right] \cdot f_{\gamma_{1}}(x) d x \\
& =1-\frac{2\left(m_{r d}-1\right) ! m_{s r}^{m_{s r}}}{\overline{\gamma_{s r}} m_{s r} \Gamma\left(m_{s r}\right) \Gamma\left(m_{r d}\right)} \exp \left(-\frac{a m_{r d} \gamma_{t h}}{\overline{\gamma_{r d}}}-\frac{m_{s r} \gamma_{t h}}{\overline{\gamma_{s r}}}\right) \\
& \times \sum_{n=0}^{m_{s r}-1} \sum_{k=0}^{m_{r d}-1} \sum_{l=0}^{k}\left(\begin{array}{l}
k \\
l
\end{array}\right)\left(\begin{array}{l}
m_{s r}-1 \\
n
\end{array}\right) a^{u}\left(a \gamma_{t h}+b\right)^{v}\left(\frac{m_{s r}}{\overline{\gamma_{s r}}}\right)^{\frac{w}{2}} \\
& \times\left(\frac{m_{r d}}{\bar{\gamma}_{r d}}\right)^{p} K_{-w}\left(2 \sqrt{\frac{m_{s r} m_{r d} \gamma_{t h}\left(a \gamma_{t h}+b\right)}{\gamma_{s r} \overline{\gamma_{r d}}}}\right) \gamma_{t h}^{q} \frac{1}{k !}
\end{aligned}
$$

where $u=k-l, v=\frac{n+l+1}{2}, w=l-n-1, p=\frac{2 k+n-l+1}{2}$, $q=\frac{2 k+2 m_{s r}-n-l-1}{2}$.

Therefore, the outage probability of system can be obtained by substituting (14), (15), and (20) into (13) as

$$
\begin{aligned}
P_{\text {out }}^{(a f)}\left(\gamma_{t h}\right) & =\exp \left(-\frac{m_{s r}}{\overline{\gamma_{s r}}} \gamma_{t h}\right) \sum_{n=0}^{m_{s r}-1}\left(\frac{m_{s r} \gamma_{t h}}{\overline{\gamma_{s r}}}\right)^{n} \frac{1}{n !} \\
& \times\left[1-\exp \left(-\frac{m_{s d}}{\overline{\gamma_{s d}}} \gamma_{t h}\right) \sum_{n=0}^{m_{s d}-1}\left(\frac{m_{s d} \gamma_{t h}}{\overline{\gamma_{s d}}}\right)^{n} \frac{1}{n !}\right] \\
& \times\left[1-\frac{2\left(m_{r d}-1\right) ! m_{s r}^{m_{s r}}}{\overline{\gamma_{s r}} m_{s r} \Gamma\left(m_{s r}\right) \Gamma\left(m_{r d}\right)} \exp \left(-\frac{a m_{r d} \gamma_{t h}}{\overline{\gamma_{r d}}}-\frac{m_{s r} \gamma_{t h}}{\overline{\gamma_{s r}}}\right)\right. \\
& \times \sum_{n=0}^{m_{s r}-1} \sum_{k=0}^{m_{r d}-1} \sum_{l=0}^{k}\left(\begin{array}{l}
k \\
l
\end{array}\right)\left(\begin{array}{l}
m_{s r}-1 \\
n
\end{array}\right) a^{u}\left(a \gamma_{t h}+b\right)^{v}\left(\frac{m_{s r}}{\overline{\gamma_{s r}}}\right)^{\frac{w}{2}} \\
& \left.\times\left(\frac{m_{r d}}{\overline{\gamma_{r d}}}\right)^{p} K_{-w}\left(2 \sqrt{\frac{m_{s r} m_{r d} \gamma_{t h}\left(a \gamma_{t h}+b\right)}{\overline{\gamma_{s r}} \bar{\gamma}_{r d}}}\right) \gamma_{t h}^{q} \frac{1}{k !}\right] \\
& +\left[1-\exp \left(-\frac{m_{s r}}{\overline{\gamma_{s r}}} \gamma_{t h}\right) \sum_{n=0}^{m_{s r}-1}\left(\frac{m_{s r} \gamma_{t h}}{\overline{\gamma_{s r}}}\right)^{n} \frac{1}{n !}\right] \\
& \times\left[1-\exp \left(-\frac{m_{s d}}{\overline{\gamma_{s d}}} \gamma_{t h}\right) \sum_{n=0}^{m_{s d}-1}\left(\frac{m_{s d} \gamma_{t h}}{\overline{\gamma_{s d}}}\right)^{n} \frac{1}{n !}\right]
\end{aligned}
$$

\section{Numerical and simulation results}

This section studies how the outage probability changes as a function of the system parameters in different situations under both AF and DF schemes. Unless otherwise explicitly specified, the main parameters adopted in our experiments and simulations are set as $P_{S}=$ $P_{R}=1, \sigma^{2}=0.01, \gamma_{t h}=0 d B, \rho=0.5$, and $E_{h 1}=E_{h 2}=E_{h 3}=1$.

In Fig. 2, the outage probability curves under DF schemes are presented where $E_{h 1}=E_{h 2}=1$ and $\rho=$ $\{0.1,0.3,0.5,0.7,0.9\}$. Clearly, the outage probability for a lower $\rho$ outperforms the one for a higher $\rho$ at the lower $E_{h 3} / E_{h 1}$ and outperforms on the contrary with the increase of the value of $E_{h 3} / E_{h 1}$. This is because a lower $\rho$ means a lower portion of the received signal power split to the ID at $R$, resulting in a higher received SNR at $R$, 


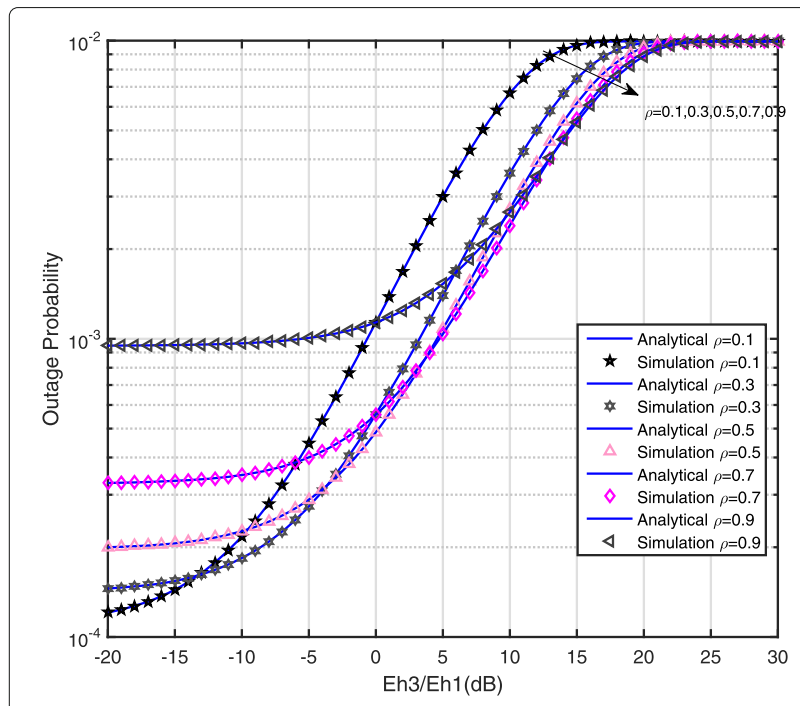

Fig. 2 Outage probability versus $E_{h 1}$ for $\rho$ with DF

which leads to a higher capacity, but with the increment of $E_{h 3} / E_{h 1}$, the relay link channel will be worse, so the outage probability will become greater when the $\rho$ increases at the same value of $E_{h 3} / E_{h 1}$. In Fig. 3, the outage probability curves under AF schemes are presented where $E_{h 1}=$ $E_{h 2}=1$ and $\rho=\{0.1,0.3,0.5,0.7,0.9\}$. We can get that the outage probability for a higher $\rho$ outperforms the one for a lower $\rho$ when $\rho$ is smaller than 0.5 and on the contrary with the increase of the value of $\rho$. This is because a lower $\rho$ means a lower portion of the received signal power split to the ID at $R$, resulting in a higher received SNR at $R$. Yet with the continuous increment of $\rho$ when $\rho$ is greater than 0.5 , the outage probability will be larger than the lower $\rho$ system at the same value of $E_{h 3} / E_{h 1}$;

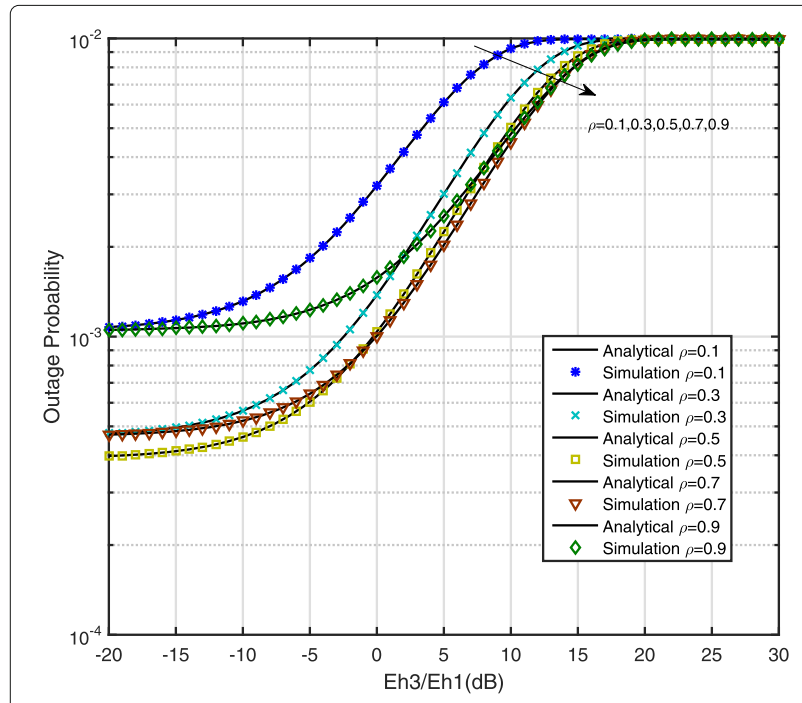

Fig. 3 Outage probability versus $E_{h 1}$ for $\rho$ with AF

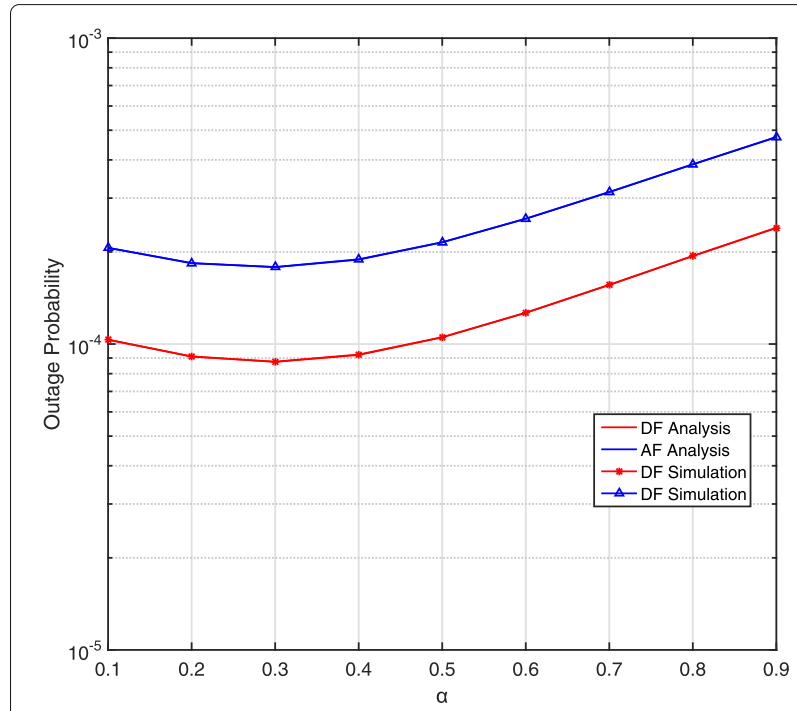

Fig. 4 Outage probability versus $d$ with DF and AF

this is because of the transmission information becoming more and more small. In Fig. 4, the outage probability curves for the disposition of $R$ between the $S$ node and the $D$ node. The best outage probability occurs at 0.3 , which is the disposition of the relay-aided node $R$ departing from $S$ node. In Fig. 5, the outage probability curves under DF and $\mathrm{AF}$ schemes are depicted where the value of $E_{\max }=$ $\{0.1,0.3,0.5,0.7,0.9\}$ and $E_{h 1}=E_{h 3}=1$. Obviously, the outage probability for a higher $E_{\max }$ outperforms that for a lower $E_{\max }$. That is to say, when the relay node harvests the more energy from ambient environment, the better system performance will be obtained.

In Figs. 6 and 7, the outage probability curves under $\mathrm{AF}$ and $\mathrm{DF}$ schemes are depicted where $E_{h 1}=E_{h 2}$ and

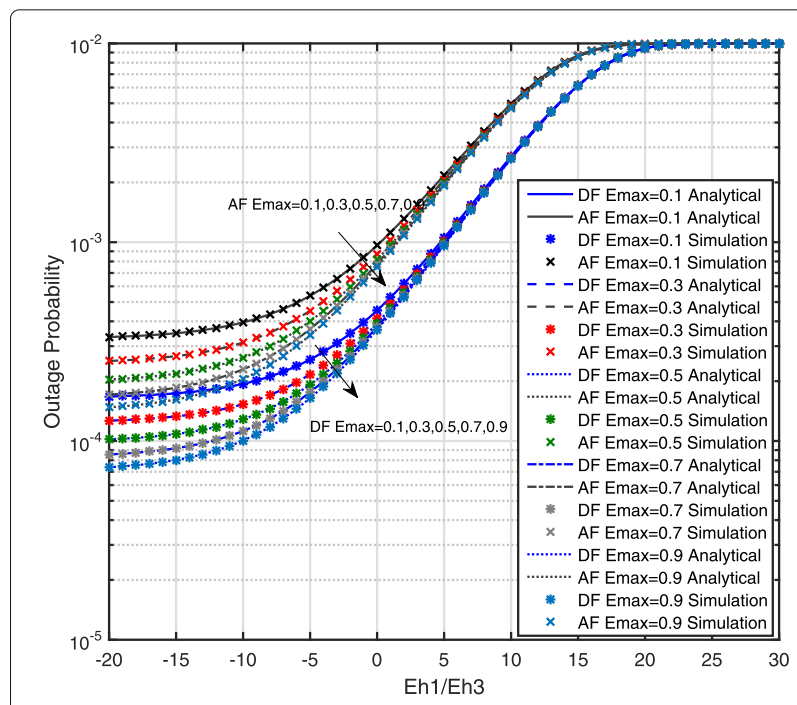

Fig. 5 Outage probability versus $E_{h 1}$ for $E_{\max }$ with $D F$ and $A F$ 


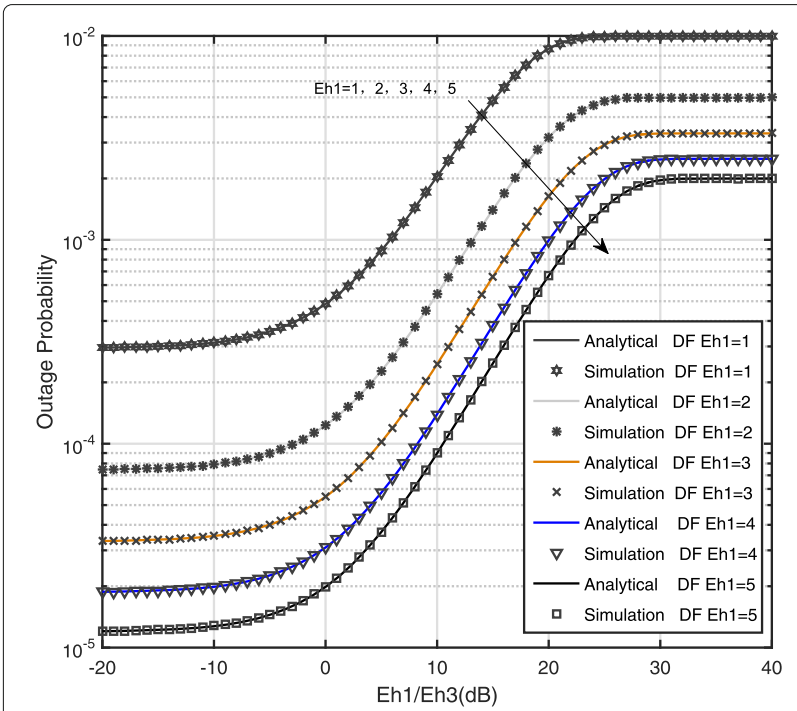

Fig. 6 Outage probability versus $E_{h 1}$ for $E_{h 2}=E_{h 3}$ with $D F$

$E_{h 1}=\{1,2,3,4,5\}$. We can get that the outage probability for a lower $E_{h 1}$ outperforms the one for a higher $E_{h 1}$. Because a lower $E_{h 1}$ means a lower probability, the SNR at each receriver falls below the threshold. It can also be observed that outage probability becomes worse when the value of $E_{h 1} / E_{h 3}$ increases. This is because the increasement of the value of $E_{h 1} / E_{h 3}$ means the worse of the $R-D$ link, resulting in an increasing outage probability over the $R-D$ link.

In Figs. 8 and 9, the outage probability curves under DF and AF schemes are depicted where $E_{h 1}=E_{h 3}=1$ and $\gamma_{t h}=\{0,1,2,3,4\}$. It is clearly that the outage probability for a lower $\gamma_{t h}$ ourperforms than that for a higher $\gamma_{t h}$.

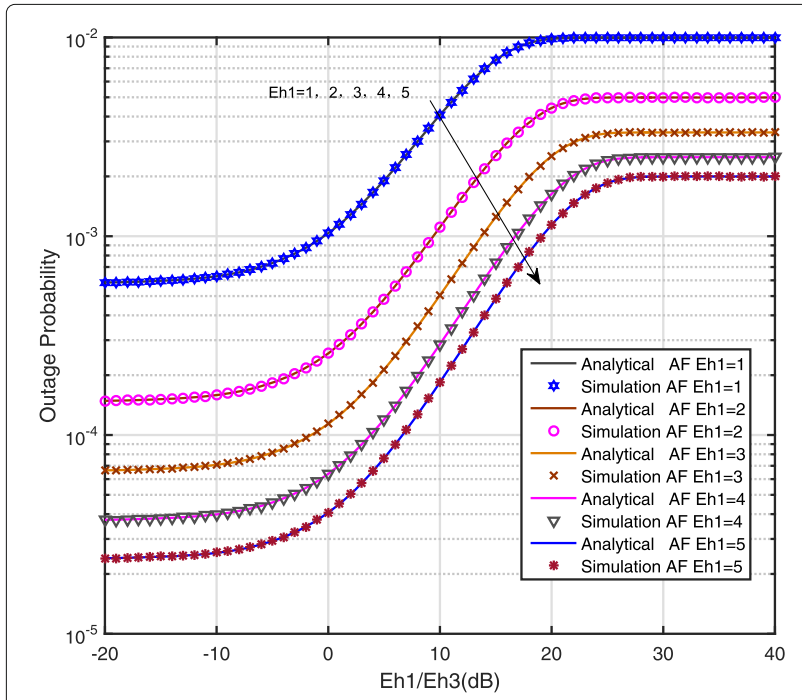

Fig. 7 Outage probability versus $E_{h 1}$ for $E_{h 2}=E_{h 3}$ with $A F$

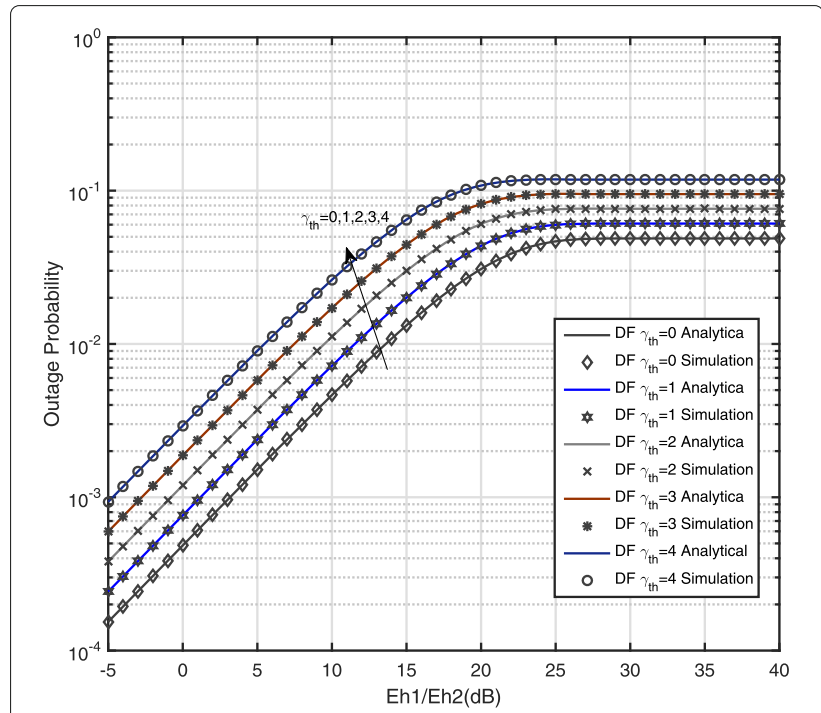

Fig. 8 Outage probability versus $\gamma_{t h}$ for $E_{h 2}=E_{h 3}$ with $D F$

Because a lower $\gamma_{t h}$ means a lower probability, the SNR at each receiver falls below the threshold. Further, outage probability becomes worse when the value of $E_{h 1} / E_{h 2}$ increases, because the increasing value of $E_{h 1} / E_{h 2}$ means the $S$ - $D$ link becoming worse too, resulting in a lower diversity gain at node $D$.

In Fig. 10, the outage probability curves under DF and $\mathrm{AF}$ schemes are depicted where $E_{h 2}=E_{h 3}=1$. It is obviously that the outage probability for a higher $m$ outperforms the one for a lower $m$. It means that the greater $m$, the more energy the relay node can receive, so the lower the probability of system interruption.

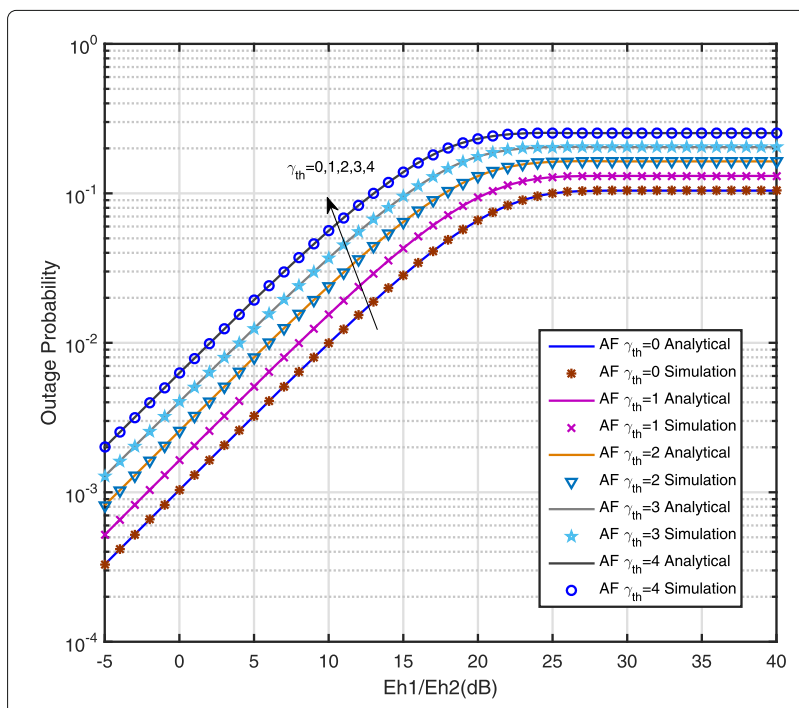

Fig. 9 Outage probability versus $\gamma_{\text {th }}$ for $E_{h 2}=E_{h 3}$ with $A F$ 


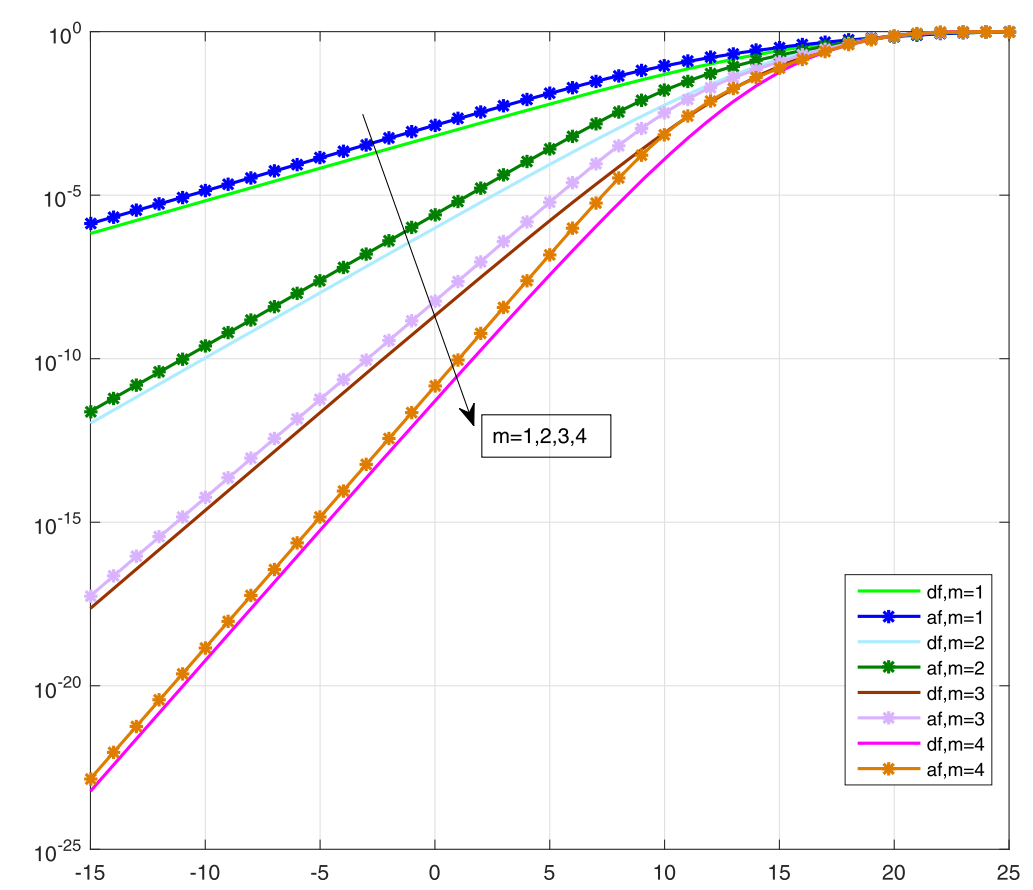

Fig. 10 Outage probability versus $m=m_{s r}=m_{r d}=m_{s d}$ for $E_{h 2}=E_{h 3}$ with DF and AF

\section{Conclusions}

In this paper, we presented the outage probability of power splitting SWIPT two-way relay networks in Nakagami$\mathrm{m}$ fading. With the help of some approximations, we got the explicit closed-form expression of outage probability of cooperative system over Nakagami-m fading. And the numerical results proposed by the present work were validated by the Monte Carlo method. From the numerical analysis and simulation results, we found that the system parameters have great impact on both AF and DF relay systems.

\section{Method}

This paper mainly studies a two-way relay SWIPT system over Nakagami-m fading, which consists of a source-destination $(S-D)$ pair and a relay node $R$ embedded power-splitting (PS). In this wireless network system, we consider the protocols of AF and $\mathrm{DF}$ and the power splitting energy receiver. In the part of experimental designment, we analyze the outage probability of the traditional energy harvesting technique, the RF energy harvesting technique, and the outage probability of this kind of relay wireless network under different parameters. At last, we verify the outage probability of the explicit closed-form expressions with the Monte Carlo statistical method. From the simulation results, we can observe that the expressions obtained in this paper are correct and effective.

\begin{abstract}
Abbreviations
$\alpha$ : The ratio of the distance between the relay node and the source node $S$ to the distance $d$ between the source node $S$ and the destination node $D ; \gamma_{r d}$ : The SNR of the received signal at information receiver of the node $D$ from the node $R_{;} \gamma_{s}$ : The SNR of the received signal at information receiver of the node $R$ from the node $S_{;} \gamma_{s d}$ : The SNR of the received signal at information receiver of the node $D$ from the node $S ; \gamma_{t h}$ : The threshold SNR; $\overline{\gamma_{r d}}$ : The average SNR of received signal at information receiver of the node $D$ from the node $R_{;} \bar{\gamma}_{s r}$ : The average SNR of received signal at information receiver of the node $R$ from the node $S_{i} \overline{\gamma_{s}}$ : The average SNR of received signal at information receiver of the node $D$ from the node $S$; $\rho$ : The ratio of power splitter; AF: Amplify-and-forward $d$ : The distance of the source node $S$ and the destination node D; DF: Decodeand-forward; EH: Energy harvesting; $E_{h 1}: E\left(\left|h_{s r}\right|^{2}\right)$, the statistical expectation of $\left|h_{s r}\right|^{2} ; E_{h 2}: E\left(\left|h_{s d}\right|^{2}\right)$, the statistical expectation of $\left|h_{s d}\right|^{2} ; E_{h 3}: E\left(\left|h_{r d}\right|^{2}\right)$, the statistical expectation of $\left|h_{r d}\right|^{2} ; E_{\text {max }}$ : The maximum natural energy harvested by relay node from ambient environment; $h_{i j}$ : The link channel gain between node $i$ and $j$; MIMO: Multiple-input multiple-out; MISO: Multiple-input single-output; NOMA: Non-orthogonal multiple access; $P_{i}$ : The transmit power at node $i ; P_{R F}$ : The energy harvested by relay node through RF; PS: Power splitting; SWIPT: Simultaneous wireless information and power transfer
\end{abstract}

\section{Acknowledgements}

The authors would like to thank the Editor and anonymous reviewers for their helpful comments and suggestions in improving the quality of this paper. This work is supported by National Natural Science Foundation general projects, China (nos. 61672217, 61304208), and the Foundation of the Science and technology project of Hunan Provincial Department of Education (no. 15C1414).

\section{Funding}

This paper was supported by the National Natural Science Foundation general projects, China (no. 61672217), by the National Natural Science Foundation general projects, China (no. 61304208), and also by the Foundation of the Science and technology project of Hunan Provincial Department of Education (no. 15C1414).

\section{Authors' contributions}

SZ has fulfilled all the system modeling, analysis, and simulation and drafted the article. RL has helped revise the manuscript. HH has given critical revision 
of the article and has helped revise the manuscript. All authors read and approved the final manuscript.

\section{Competing interests}

The authors declare that they have no competing interests.

\section{Publisher's Note}

Springer Nature remains neutral with regard to jurisdictional claims in published maps and institutional affiliations.

\section{Author details}

${ }^{1}$ College of Computer Science and Electronic Engineering and the Key Laboratory for Embedded and Network Computing, Hunan University, 410082 Changsha, People's Republic of China. ${ }^{2}$ School of Computer and Information Engineering, Central South University of Forest and Technology, Changsha 410004, People's Republic of China.

Received: 4 August 2017 Accepted: 5 December 2017

Published online: 10 January 2018

\section{References}

1. C Huang, R Zhang, S Cui, Throughput maximization for the gaussian relay channel with energy harvesting constraints. IEEE J. Sel. Areas Commun. 31(8), 1469-1479 (2013)

2. WKG Seah, ZA Eu, HP Tan, in 2009 1st International Conference on Wireless Communication Vehicular Technology Information Theory and Aerospace Electronic Systems Technology. Wireless sensor networks powered by ambient energy harvesting (WSN-HEAP) survey and challenges, (2009), pp. $1-5$

3. R Jiang, K Xiong, P Fan, Y Zhang, Z Zhong, Optimal design of SWIPT systems with multiple heterogeneous users under non-linear energy harvesting model. IEEE Access. 5(5), 11479-11489 (2017)

4. KXiong, P Fan, T Li, KB Letaief, Rate-energy region of SWIPT for MIMO broadcasting under nonlinear energy harvesting model. IEEE Trans. Wirel. Commun. 16(8), 5147-5161 (2017)

5. L Varshney, in Proc. IEEE Int. Symp. Inf. Theory. Transporting Information and Energy Simultaneously, (2008), pp. 1612-1616

6. X Zhou, R Zhang, CK Ho, Wireless information and power transfer: architecture design and rate-energy tradeoff. IEEE Trans. Commun. 61(11), 4754-4767 (2013)

7. Y Zhang, KXiong, P Fan, HC Yang, X Zhou, Space-time network coding with multiple AF relays over Nakagami- $m$ fading channels. IEEE Trans. Veh. Technol. 66(7), 6026-6036 (2017)

8. KXiong, P Fan, Y Lu, KB Letaief, Energy efficiency with proportional rate fairness in multirelay OFDM networks. IEEE J Sel. Areas Commun. 34(5), $1431-1447$ (2016)

9. J Liu, KXiong, P Fan, Z Zhong, RF energy harvesting wireless powered sensor networks for smart cities. IEEE Access. 5, 9348-9358 (2017)

10. KXiong, P Fan, HC Yang, KB Letaief, Space-time network coding with overhearing relays. IEEE Trans. Wirel. Commun. 13(7), 3567-3582 (2014)

11. R Jiang, K Xiong, P Fan, $Y$ Zhang, Z Zhong, Outage analysis and optimization of SWIPT in network-coded two-way relay networks. Mobile Inf. Syst. 2017, (2017)

12. AA Nasir, X Zhou, S Durrani, RA Kennedy, Relaying protocols for wireless energy harvesting and information processing. IEEE Trans. Wireless Commun. 12(7), 3622-3636 (2013)

13. G Pan, C Tang, Outage performance on threshold AF and DF relaying schemes in simultaneous wireless information and power transfer systems. Int. J. Electron. Commun. 71, 175-180 (2017)

14. A Ikhlef, MZ Bocus, in IEEE Wireless Communications and Networking Conference, WCNC 2016. Outage performance analysis of relay selection in SWIPT systems, (Doha, Qatar, 2016), pp. 1-5

15. X Di, KXiong, P Fan, HC Yang, Simultaneous wireless information and power transfer in cooperative relay networks with rateless codes. IEEE Trans. Veh. Technol. 66(4), 2981-2996 (2017)

16. KXiong, P Fan, T Li, KB Letaief, Group cooperation with optimal resource allocation in wireless powered communication networks. IEEE Trans. Wirel. Commun. 16(6), 3840-3853 (2017)

17. Z Ding, SM Perlaza, I Esnaola, HV Poor, Power allocation strategies in energy harvesting wireless cooperative networks. IEEE Trans. Wirel. Commun. 13(2), 846-860 (2014)
18. Z Yang, Z Ding, P Fan, N Al-Dhahir, The impact of power allocation on cooperative non-orthogonal multiple access networks with SWIPT. IEEE Trans. Wirel. Commun. 16(7), 4332-4343 (2017)

19. K Xiong, P Fan, Z Xu, HC Yang, KB Letaief, Optimal cooperative beamforming design for MIMO decode-and-forward relay channels. IEEE Trans. Signal Process. 62(6), 1476-61489 (2014)

20. Y Liu, Joint resource allocation in SWIPT-based multi-antenna decode-and-forward relay networks. IEEE Trans. Veh. Technol. 66(10), 9192-9200 (2017)

21. KXiong, P Fan, T Li, KB Letaief, Outage probability of space-time network coding over Rayleigh fading channels. IEEE Trans. Veh. Technol. 63(4), 1965-1970 (2014)

22. KXiong, P Fan, C Zhang, KB Letaief, Wireless information and energy transfer for two-hop non-regenerative MIMO-OFDM relay networks. IEEE J. Sel. Areas Commun. 33(8), 1595-1611 (2015)

23. J Tang, DKC So, A Shojaeifard, KK Wong, J Wen, Joint antenna selection and spatial switching for energy effcient MIMO SWIPT system. IEEE Trans. Wirel. Commun. 16(7), 4751-4769 (2017)

24. KXiong, P Fan, C Zhang, KB Letaief, Wireless information and energy transfer for two-hop non-regenerative MIMO-OFDM relay networks. IEEE J. Sel. Areas Commun. 33(8), 1595-1611 (2015)

25. R Feng, Q Li, Q Zhang, J Qin, Robust secure transmission in MISO simultaneous wireless information and power transfer system. IEEE Trans. Veh. Technol. 64(1), 400-405 (2015)

26. G Pan, H Lei, Y Deng, L Fan, J Yang, Y Chen, Z Ding, On secrecy performance of MISO SWIPT systems with TAS and imperfect CSI. IEEE Trans. Commun. 64(9), 3831-3843 (2016)

27. IS Gradshteyn, IM Ryzhik, Tables of integrals, series, and products, 7th Ed, (2007)

28. A Molisch, AF Molisch, Wireless communications (2005)

29. A Goldsmith, A Nin, Wireless communications (2005)

\section{Submit your manuscript to a SpringerOpen ${ }^{\circ}$ journal and benefit from:}

- Convenient online submission

Rigorous peer review

- Open access: articles freely available online

- High visibility within the field

- Retaining the copyright to your article

Submit your next manuscript at $>$ springeropen.com 